\title{
Modeling of Efficient Circular Polarized Rectenna Design for Microwave Power Transmission at $2.45 \mathrm{GHz}$
} \author{
Hamid Bennis ${ }^{2}$, Mohamed Latrach ${ }^{4}$ \\ ${ }^{1}$ LITEN Laboratory FPK - Khouribga /FSTS, University of Hassan the $1^{\text {ST }}$ Settat, Morocco \\ ${ }^{2}$ LMEET Laboratory FPK - Khouribga /FSTS, University of Hassan the $1^{\text {ST }}$ Settat, Morocco \\ ${ }^{3}$ National Institute of Post and Telecommunication (INPT), Rabat, Morocco \\ ${ }^{4}$ RF \& Hyper group ESEO, Angers, France
}

Mohamed Adel Sennouni ${ }^{1, ~ *, ~ J a m a l ~ Z b i t o u ~}{ }^{2}$, Benaissa Abboud ${ }^{1}$, Abdelwahed Tribak $^{3}$,

Email address:

adelsennouni@gmail.com (M. A. Sennouni)

\section{To cite this article:}

Mohamed Adel Sennouni, Jamal Zbitou, Benaissa Abboud, Abdelwahed Tribak, Hamid Bennis, Mohamed Latrach. Modeling of Efficient Circular Polarized Rectenna Design for Microwave Power Transmission at 2.45GHz. American Journal of Electromagnetics and Applications. Vol. 3, No. 4, 2015, pp. 24-30. doi: 10.11648/j.ajea.20150304.11

\begin{abstract}
This paper presents a planar rectenna (rectifier + antenna) design at $2.45 \mathrm{GHz}$ ISM band for microwave power transmission for powering devices involving low power consumption, located in unreachable area or needing expensive maintenance costs. The proposed design incorporate a $3 \times 3$ circular polarized antenna array with an enhanced directivity of $9.14 \mathrm{dBi}$ and an overall size of $26.18 \times 11.86 \mathrm{~cm}^{2}$ modeled by using CST Microwave Studio software, and associated to a microstrip rectifier based on a voltage doubler circuit with HSMS 2822 Schottky diode designed and simulated by using schematic simulation in ADS. A high conversion efficiency of $71 \%$ and a DC output voltage of $10 \mathrm{~V}$ can be reached for a given input power level of $20 \mathrm{dBm}$ with an optimum load of $1500 \Omega$.
\end{abstract}

Keywords: Microwave Power Transmission, Rectenna, Antenna Array, Circular Polarization, RF-to-DC Microstrip Rectifier

\section{Introduction}

Over 100 years ago, the concept of Wireless Power Transmission (WPT) that was theorized, developed and demonstrated by Nikola Tesla in the early 1900s, have known a great advance and successful impact in many real life applications. Then it has been a topic of continued interests since the last several decades. Wireless power transmission is the transmission of electrical energy from a power source to an electrical load without wired connections. It is useful in cases where interconnecting wires are inconvenient, hazardous, or impossible. In particular, a WPT system converts direct current (DC) power to microwaves, transmits that microwave radiation in the free space to a target. Once DC power is converted to microwaves and transmitted into space, a system that requires power needs to receive and convert microwaves back to useful DC power. The device used for this reception and conversion is called a rectenna (rectifying antenna), this term is derived from the fact that a rectenna is comprised of an antenna coupled with a rectifying circuit. The rectenna device was created by W.C. Brown in his groundbreaking work in WPT in the 1960s in Raytheon Technology Company in USA. He demonstrated its ability in 1964 by powering a helicopter from the solely through microwaves. Obviously, WPT could revolutionize our energy sector, our information and communication technology sector, our environment sector, and our medical sector. First, the proposed SPS (Solar Power Satellite) would bring clean energy from natural sunlight in space to power Earth without using the resources of our planet. This could fundamentally change the landscape of resource planning and energy renewable strategies. This idea was first proposed in 1968 and all of the experiments have only been carried out in terrestrial laboratories. The SPS satellites would be put in high earth orbit at geosynchronous location. This would allow them to receive light $99 \%$ of the year.

Hence the development of efficient rectifying antennas (rectennas) has become a hot and vital research topic [1]. In fact, a rectenna representing the key element for EM "Electromagnetic" energy harvesting and Wireless Power Transmission applications, thus device designed to collect the energy associated to a free propagating EM wave and to 
transform it into Direct Current (DC) power. Recently, several rectenna devices have been proposed in the literature investigating several operating frequencies [2-4].Components of microwave power transmission have traditionally been focused on $2.45 \mathrm{GHz}$ and recently moving up to $5.8 \mathrm{GHz}$, which has a smaller antenna aperture area than that of 2.45 $\mathrm{GHz}$. Further both frequencies have comparably low atmospheric loss, cheap components availability, and reported high conversion efficiency.

A block diagram of the basic architecture of a rectenna is illustrated in Fig.1. The Radio Frequency (RF)/microwave EM energy is collected by an antenna and converted into DC power by a rectifying circuit (the rectifier). In order to improve the rectenna RF-to-DC conversion two blocks can be added respectively between the antenna and the rectifier, also between the rectifier and the load resistor, in addition it's necessary to further reduce the transmission loss and to increase the input voltage of the rectifier circuit. These blocks act both as filtering and as matching sections, they should be optimized in order to simultaneously fulfill the following functions:

- to match the antenna and the load to the rectifier;

- to preserve the antenna from re-irradiating the high order harmonics generated by the rectifier must be filtered;

- to preserve the load from any RF signals which are high order harmonics generated by the Schottky diode (to this end the block between the rectifier and the load should be a DC pass filter[5-6].

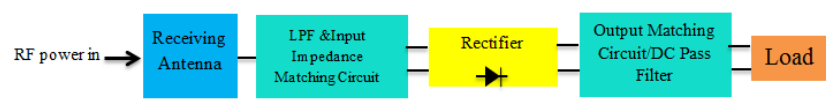

Figure 1. The block diagram of rectenna.

Recently, many kinds of rectenna were reported, including linear [7-10] and circular polarization [11-15]. The advantage of a circularly polarized (CP) rectenna over a linearly polarized one is that nearly constant DC output can be achieved and significant level of power can be reached at the reception of the rectenna. However most of the cited works and others existing in the literature incorporate a single antenna or antenna array with linear polarization, furthermore the researches focus to interconnect many rectennas in order to achieve high DC output (power and/or voltage), and as a result the size of the global system can be increased, which makes it difficult to integrate in an electronic system such as wireless sensors and mobile phones.

In this paper we are going to present a new rectenna design with high performances for microwave power transmission (MPT). The developed rectenna is aimed to capturing the RF energy from the free space at the ISM frequency band of 2.45 GHz. In fact it's formed by a receiving antenna which incorporates a new antenna array with circular polarization and a RF-to-DC microstrip rectifier is constructed from a single stage voltage doubler circuit with Schottky diode HSMS2822, and output DC pass filter associated with the load resistor. An output voltage of $10 \mathrm{~V}$ and RF-to-DC conversion efficiency of more than $71 \%$ can be achieved for an input RF power level of $20 \mathrm{dBm}$ with an optimum load of $1.5 \mathrm{k} \Omega$.

\section{Antenna Array Design}

In rectenna application, it is suitable to design antennas with very high directive characteristics to meet the demands of long-distance links. Hence the aim is to use the rectenna to transfer DC power through wireless links for a long distance, this can be only accomplished by increasing the electrical size of the antenna. We present in this section the antenna array design which consisting of a $3 \times 3$ square patch antenna etched on FR4 substrate $\left(\varepsilon_{\mathrm{r}}=4.4\right.$, thickness $=1.58 \mathrm{~mm}, \tan \delta$ $=0.025)$, in each antenna elements we have applied two techniques, the first one is an inclined slot at the center to obtain the circular polarization, we have chosen this technique in order to avoid the increase or the modification of the antenna size, the second one is a V shaped slots at the corners of each antenna in order to increase the bandwidth around the operating frequency of $2.45 \mathrm{GHz}$. Fig.2 presents the geometry of the developed antenna array.

This new arrangement can minimize the effects of mutual coupling between the array elements and can avoid the generation of higher modes; furthermore it can occupy a reduced area for such an antenna array of the same number of elements [16]. Firstly we have optimized the antenna dimensions and after the final antenna array structure to obtain a resonance frequency at $2.45 \mathrm{GHz}$. Optimizations were performed by using the full-wave simulator CST Microwave Studio [17] and Table.1 below shows the various optimized parameters of the proposed antenna array. Also the characteristic impedances of the microstrip lines have been used for feeding various elements of the array are given in Table 2 .

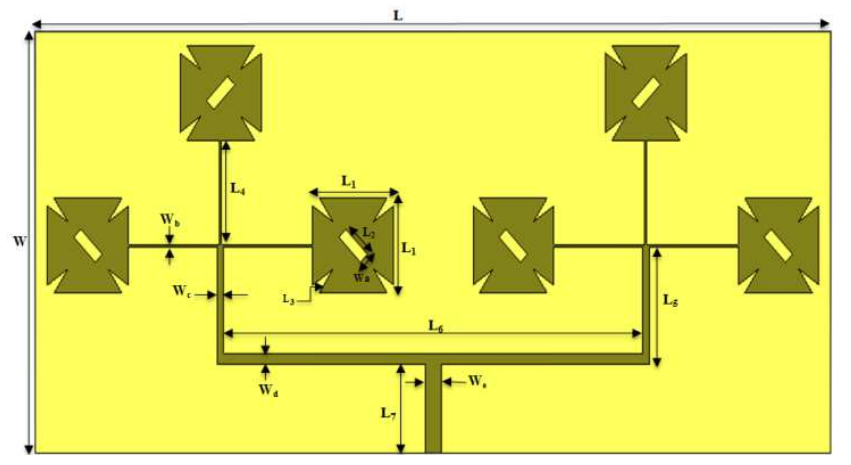

Figure 2. Geometry of proposed $3 \times 3$ antenna array.

Table 1. Physical Dimensions of the $3 \times 3$ Antenna Array.

\begin{tabular}{llll}
\hline Parameters & Value $(\mathbf{m m})$ & Parameters & Value $(\mathbf{m m})$ \\
\hline $\mathrm{W}$ & 118.6 & $\mathrm{~L}_{1}$ & 26.52 \\
$\mathrm{~W}_{\mathrm{a}}$ & 3.3 & $\mathrm{~L}_{2}$ & 10 \\
$\mathrm{~W}_{\mathrm{b}}$ & 0.704 & $\mathrm{~L}_{3}$ & 8 \\
$\mathrm{~W}_{\mathrm{c}}$ & 2.123 & $\mathrm{~L}_{4}$ & 29.29 \\
$\mathrm{~W}_{\mathrm{d}}$ & 3.044 & $\mathrm{~L}_{5}$ & 33.04 \\
$\mathrm{We}$ & 5.12 & $\mathrm{~L}_{6}$ & 137.87 \\
$\mathrm{~L}$ & 261.8 & $\mathrm{~L}_{7}$ & 33.04 \\
\hline
\end{tabular}


Table 2. Microstrip Line Impedances.

\begin{tabular}{lllll}
\hline Microstrip Line Impedance & $\mathbf{Z}_{\mathbf{b}}$ & $\mathbf{Z}_{\mathbf{c}}$ & $\mathbf{Z}_{\mathbf{d}}$ & $\mathbf{Z}_{\mathrm{e}}$ \\
\hline Value $(\Omega)$ & 100 & 61.237 & 50 & 35.35 \\
\hline
\end{tabular}

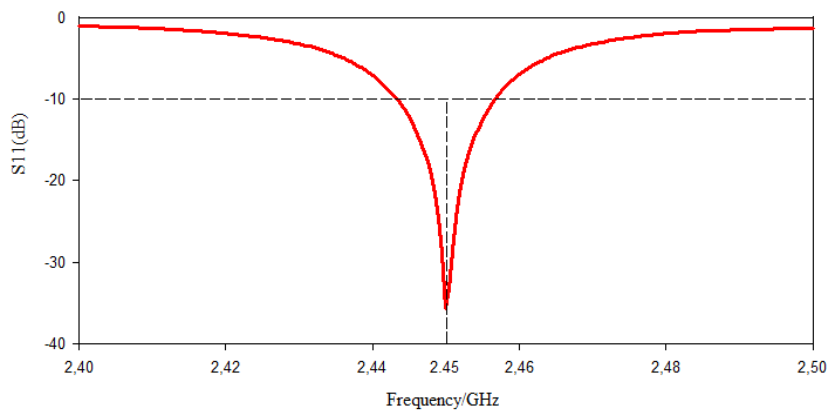

Figure 3. Simulation results of the return loss@2.45 GHz of the CP antenna array.

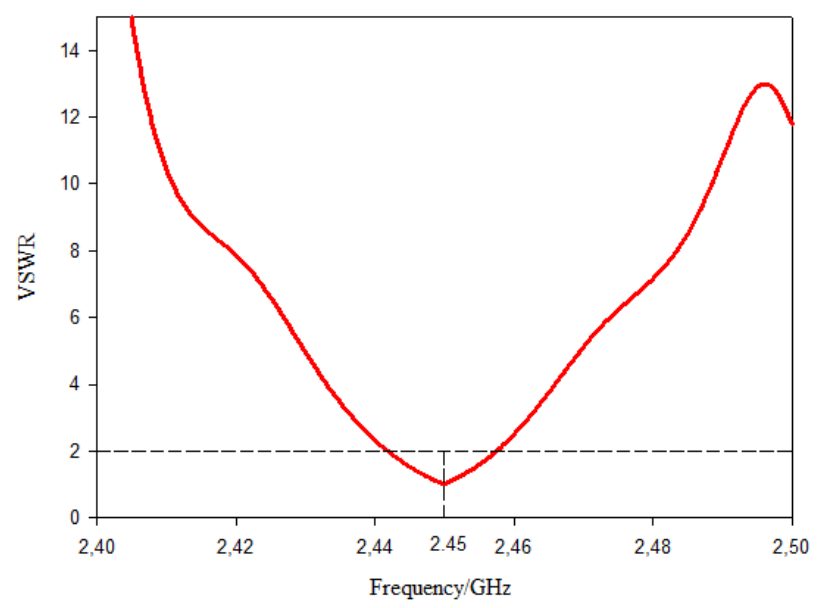

Figure 4. Simulated VSWR @ 2.45 GHz of the CP antenna array. loss, the voltage standing wave ratio (VSWR) also the axial ratio and the directivity of the developed array are evaluated. It can be noticed that this performances are greatly affected by the slots size and distance between the various patches also by the feeding lines chosen to match all elements at the resonant frequency. Figures 3 and 4 depict the simulated return loss and VSWR of the presented CP antenna array.

From the graphs it is clear that the reflection coefficient $(|\mathrm{S} 11|)(\mathrm{dB})$ of the proposed antenna array provides a good matching input impedance with a good return loss of $37.48 \mathrm{~dB}$ and VSWR of 1.02 at operating frequency of 2.45 $\mathrm{GHz}$, and having a bandwidth of $16 \mathrm{MHz}$ which is from 2.441 to $2.457 \mathrm{GHz}$. In addition to achieve a CP operation, the axial ratio has to be kept below $3 \mathrm{~dB}$. Eventually the optimum values of the proposed antenna array parameters give a better axial ratio with a minimum of $1.36 \mathrm{~dB}$ at operation frequency of $2.45 \mathrm{GHz}$ as shown in Fig. 5 .

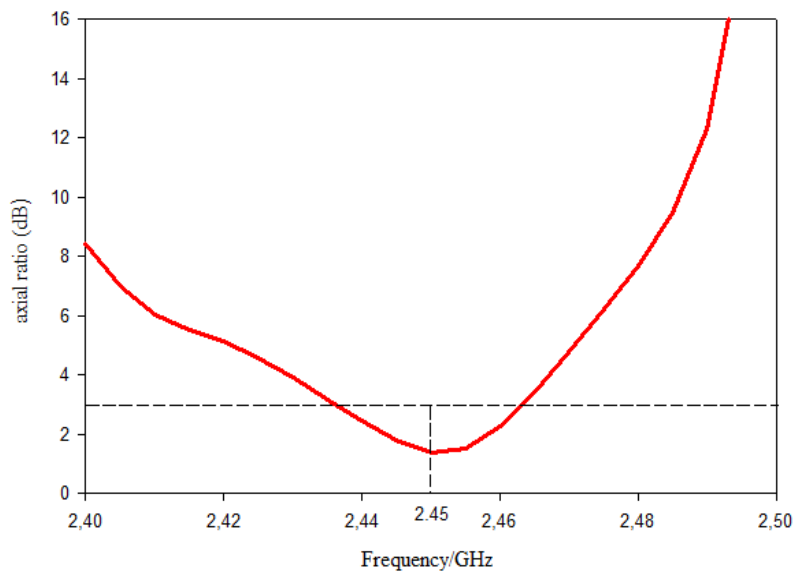

Figure 5. Simulated Axial Ratio (AR)@2.45 GHz of the CP antenna array.

The performances of the $\mathrm{CP}$ antenna array, such as return

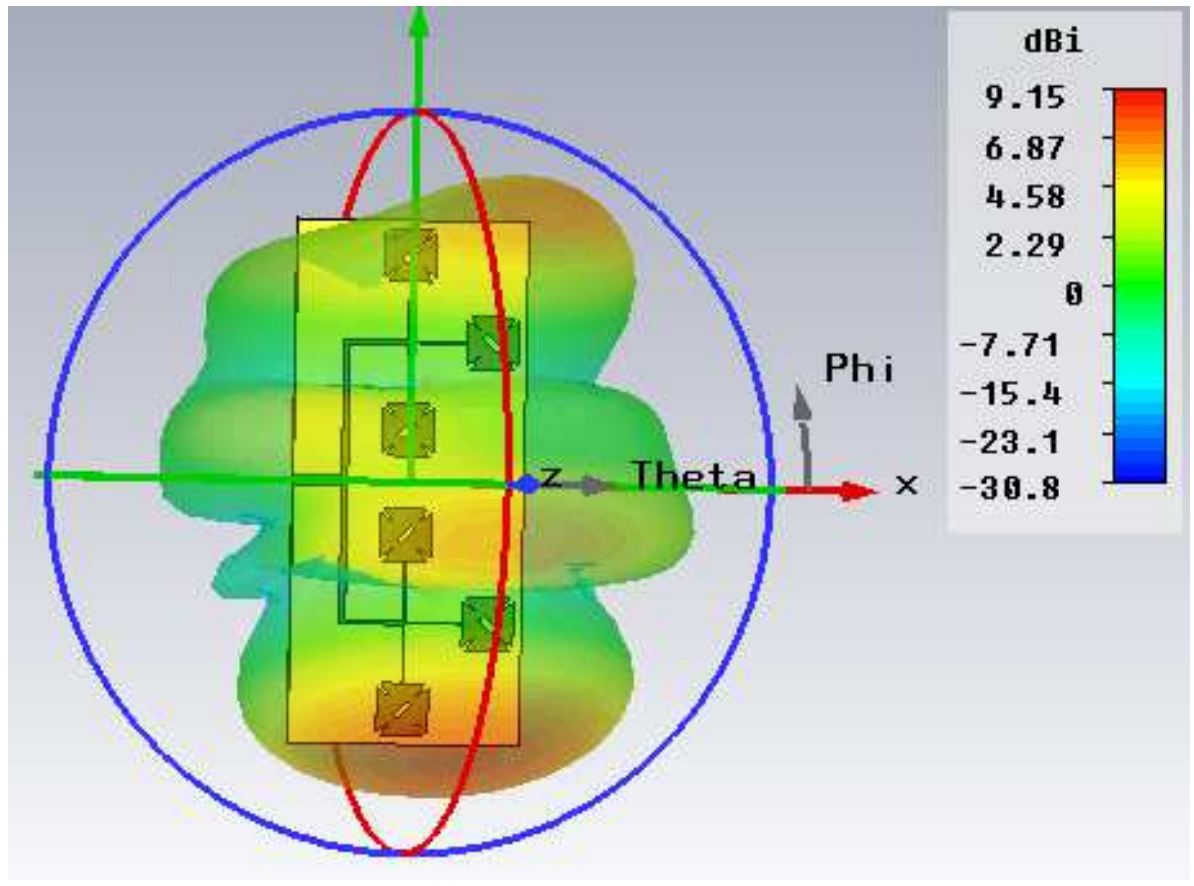


Farfield Directivity Abs (Phi=0)

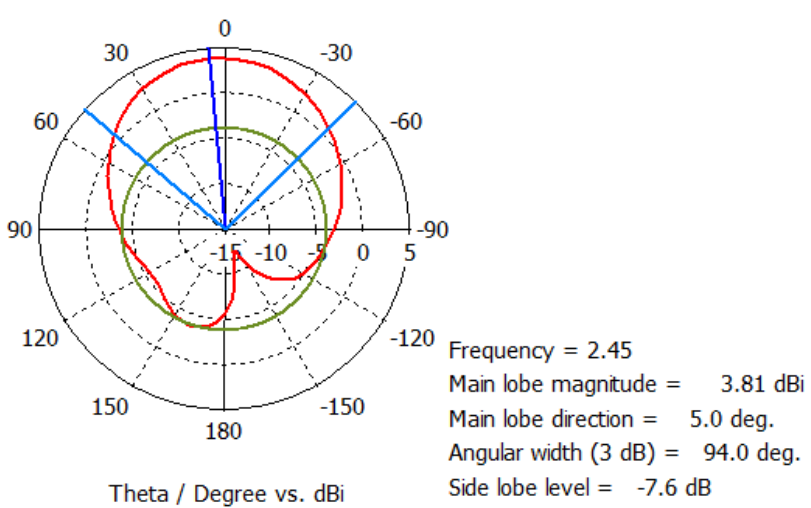

(a)
Farfield Directivity Abs (Phi=90)

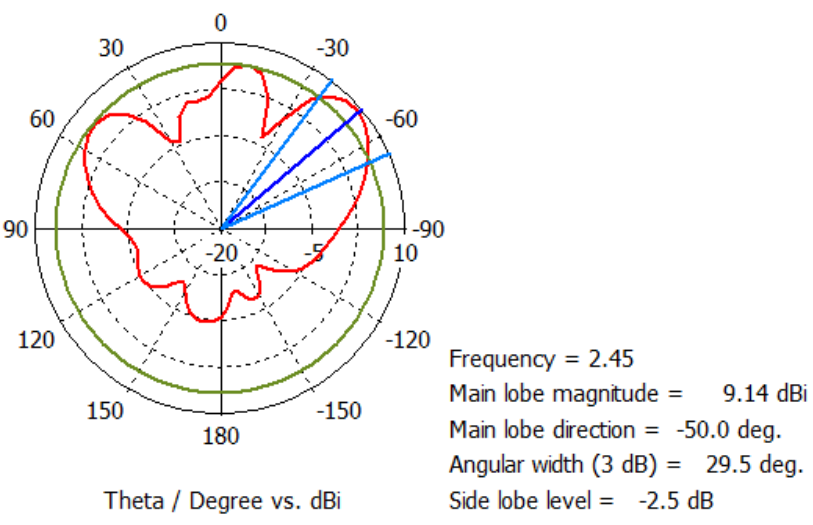

(b)

Figure 6. Three and Two-dimensional radiation pattern (a) E-plane, (b) H-plane of the CP antenna array@2.45 GHz.

Hence the radiation pattern is taken into account to predict the capabilities of the developed array to harvest microwave power. Fig.6 shows the simulated radiation pattern for the developed CP antenna array in E-plane and H-plane at operating frequency of $2.45 \mathrm{GHz}$. The graphs shows that the antenna provides a directional behavior with a half power beam width of $94^{\circ}$ and $29.8^{\circ}$ for E-plane and H-plane respectively, with enhanced directivity of about $9.14 \mathrm{dBi}$ is achieved.

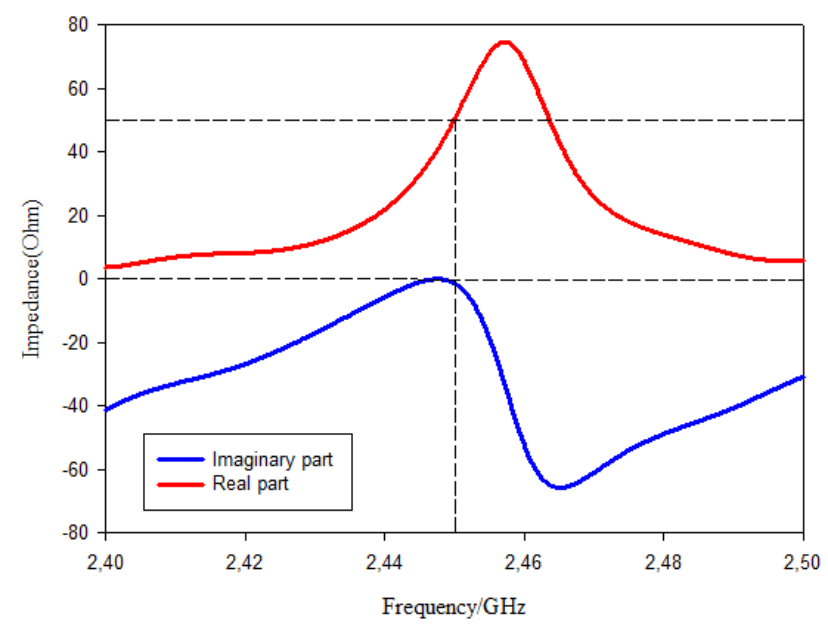

Figure 7. Simulated input antenna impedance (Ohm) vs. frequency of the proposed CP antenna array.

In Fig. 7, the simulated variation of the antenna input impedance versus frequency of the $3 \times 3 \mathrm{CP}$ antenna array can be seen. At the operating frequency of $2.45 \mathrm{GHz}$, the average value of the resistance (real part) is $50 \mathrm{Ohms}$ and the average value of the reactance (imaginary part) is 0 -Ohms which gives the adequate input impedance matching at the desired resonant frequency. Also Fig. 8 depicts the simulated input power versus frequency. The input power available at the antenna port is 0.5 Watt, hence the radiated power can reach a maximum value of about 0.49 Watt, however the reflected one has a minimum value of approximately 0 Watt at 2.445 $\mathrm{GHz}$, which means that the previous developed antenna array has the required potential to be used in a rectenna system with a minimum power loss. Furthermore Fig.9 presents the simulated antenna radiation efficiency versus frequency of the developed CP $3 \times 3$ antenna array. From the graph it's clear that the antenna radiation efficiency has a peak efficiency of $98 \%$ at $2.445 \mathrm{GHz}$.

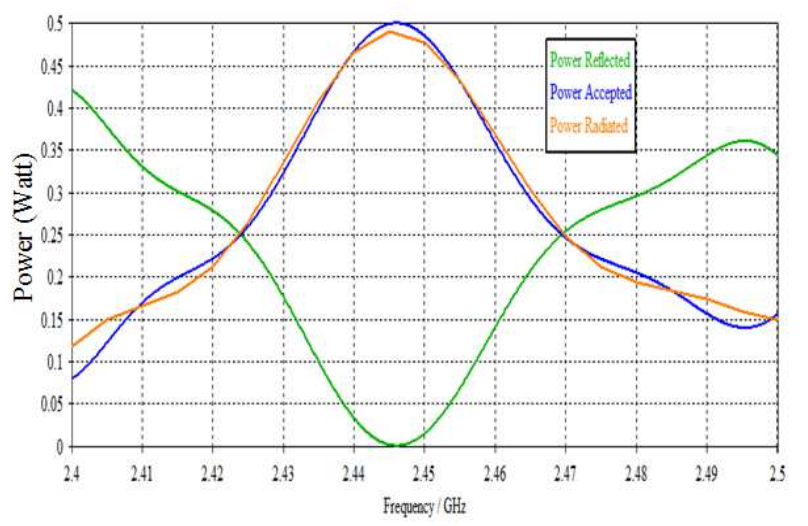

Figure 8. Simulated powers at input antenna array port (Watt) vs. frequency.

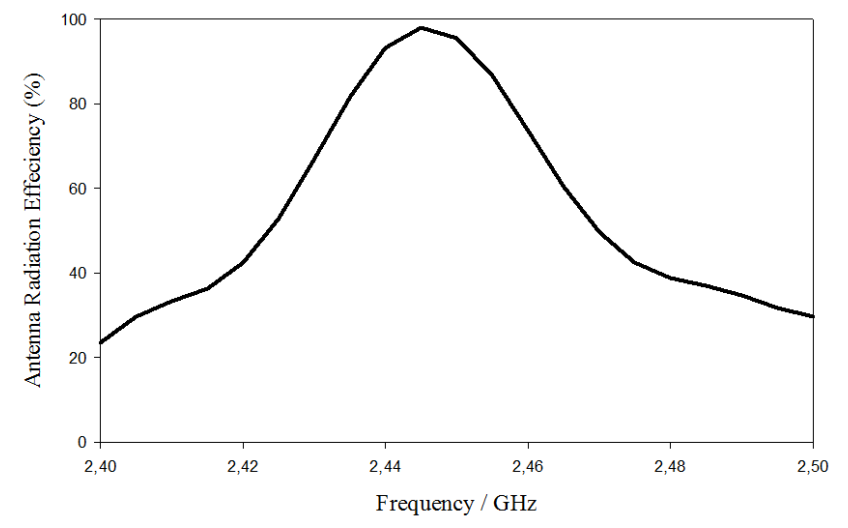

Figure 9. Simulated Antenna Radiation Efficiency vs. frequency of the proposed CP antenna array.

Consequently it is clear from the above results and discussion that the proposed design has the required performances and the potential to be investigated in a rectenna system. 
Table 3. Overview of antenna array at $2.45 \mathrm{GHz}$ described in the literature for rectenna system.

\begin{tabular}{lll}
\hline Ref & Antenna type & Antenna gain \\
\hline$[7]$ & LP 4 element patch array & $7 \mathrm{dBi}$ \\
{$[26]$} & CP multilayers 4 circular patch array & $10.8 \mathrm{dBi}$ \\
{$[27]$} & CP 4 element patch array with reflector & $6.56 \mathrm{~dB}$ \\
This work & CP 6 element patch array & $9.14 \mathrm{dBi}$ \\
\hline
\end{tabular}

\section{2.45 GHz Microstrip Rectifier Design}

The microwave rectifier can take several configurations. However, the single serial [8-18-19] and shunt configuration [10-15-20] are the most used. In addition to improve the output de voltage, a voltage doubler [21-22-23] can also be used. Then in order to increase the conversion efficiency and simplify the full-wave rectifier circuit we have used a voltage doubler for rectifying circuit etched on the same FR4 substrate used for designing the receiving antenna. As we can see in the layout of the proposed rectifier design presented in Fig. 10, it consist at least of three essential components: a single stage voltage doubler circuit based on a Zero-Bias Schottky diode HSMS 2822 [24], is associated with an input matching circuit having a form of a T-microstrip line with short end which can match the input impedance of the circuit to $50 \Omega$, and an output DC pass filter. Then this rectifier circuit can significantly increase the input voltage by using the first capacitor which charges at the negative half wave and discharges at the positive half wave. Also a low pass filter which contains two folded quarter wavelength open stubs and a T-shape and having a cutoff frequency of 1.7 $\mathrm{GHz}$ is applied before the resistive load to block the harmonic frequencies produced in rectifying operation as shown in Fig.11.

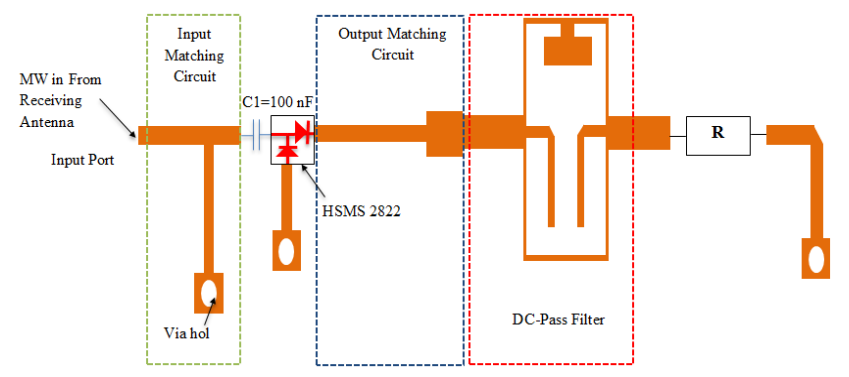

Figure 10. Layout of the Proposed RF-to-DC Microwave Rectifier.

In order to accurately predict the circuit performances and DC output an analysis approach by using ADS software is applied to simulate the schematic of the rectifier, and analyze the rectifying efficiency. By using Harmonic Balance Simulation and Large Signal Scattering Simulation in ADS [25], the nonlinear behavior of rectifier at different incident power can be observed. The choice of load resistance was done to improve and to get the best value of the conversion efficiency and also the maximum DC voltage delivered to the load.

The simulated return loss (S11) of the proposed rectifier versus the input power is presented in Fig.12. The results shows that the proposed rectifier is well matched at $2.45 \mathrm{GHz}$ $(\mathrm{S} 11<-25 \mathrm{~dB})$ around $5 \mathrm{dBm}$ input power, and a good return loss less than $-15 \mathrm{~dB}$ is obtained from about -3 to $28 \mathrm{dBm}$.

Then the conversion efficiency is an important parameter for such rectifier to predict its ability to be used for RF-toDC microwave conversion. Due to the characteristics of the diode the input power level is one of the main factors that can affect the rectifier conversion efficiency which $(\eta)$ is defined as follows:

$$
\eta_{R F-D C}=\frac{P_{D C}}{P_{\text {Recived }}} \times 100 \%
$$

Where $\mathrm{P}_{\mathrm{DC}}$ is the $\mathrm{DC}$ power produced at the load resistance $R$ of the rectenna and $P_{\text {received }}$ is power received at the antenna, which can be calculated from the Friis transmission equation (Equation 2).

$$
P_{\text {Received }}=\left(\frac{\lambda}{4 \pi r}\right)^{2} P_{t} G_{t} G_{r}
$$

Where, $G_{r}$ is the gain of the receiving antenna, $\lambda$ is the operating wavelength, $G_{t}$ and $P_{t}$ are the gain and transmitted power of the transmitting antenna, and $\mathrm{r}$ is the propagation distance.

The computed RF-to-DC conversion efficiency ( $\eta$ ) as function of input power from -30 to $30 \mathrm{dBm}$ for three resistive loads is demonstrated in Fig.13. Due to the higher breakdown voltage $(\mathrm{BV}=15 \mathrm{~V})$ of the HSMS 2822 diode used in the microstrip rectifier design, it is clear from the above results that the proposed rectifier can rectify high input powers. To this regard, it can be also noticed that the conversion efficiency increases with the increment of incident power from about $-10 \mathrm{dBm}$ and can reach a maximum value of $71 \%$ at $20 \mathrm{dBm}$ when the resistive load has $1500 \Omega$. Though it decrease when the value of the resistive load is increased. Moreover the efficiency varies slightly over a wide range of input power between 18 to 25 $\mathrm{dBm}$. In addition with the same resistive load, the DC output voltage can reach $10 \mathrm{~V}$ and more than $18 \mathrm{~V}$ at $20 \mathrm{dBm}$ and 25 $\mathrm{dBm}$ respectively (Fig.14).

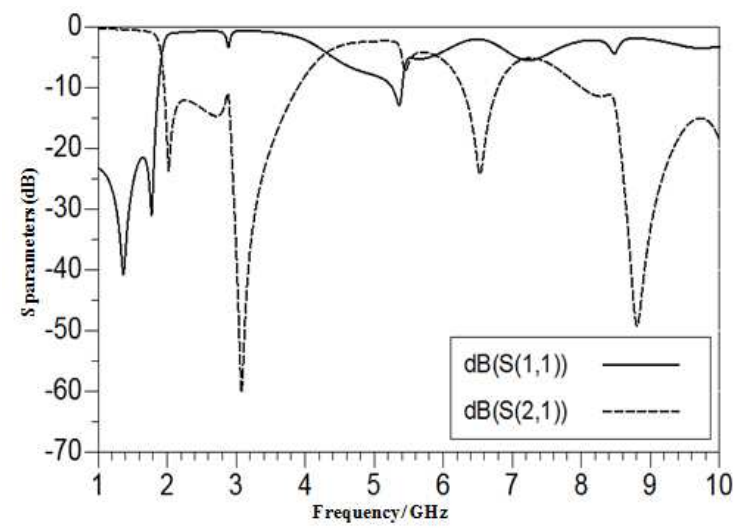

Figure 11. Simulation Results of S-Parameter responses of the DC-LPF obtained by using Agilent ADS. 


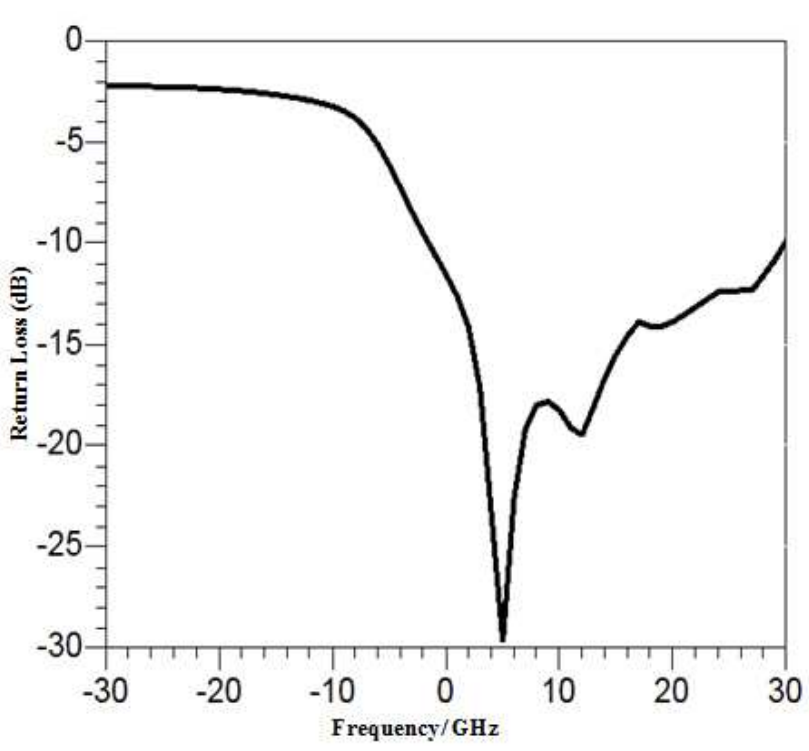

(a)

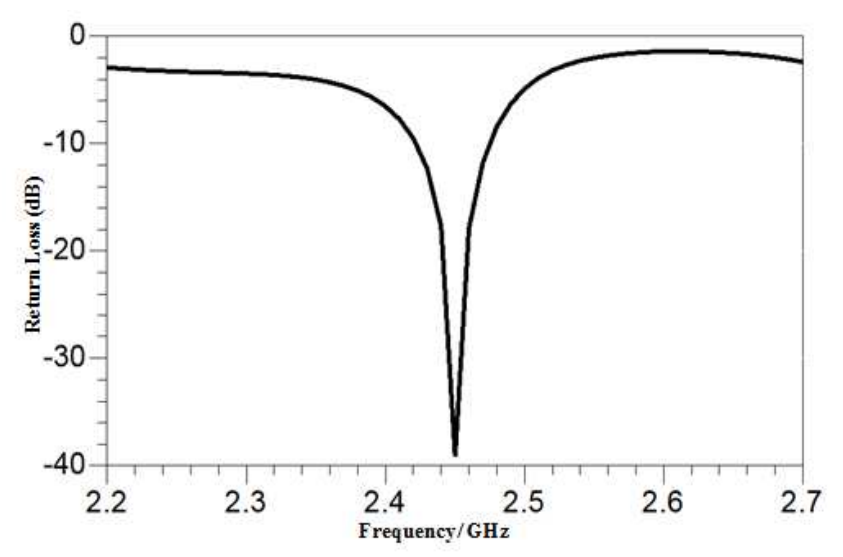

(b)

Figure 12. Return loss of the proposed rectifier (a): versus input power (HB simulation), (b): versus frequency.

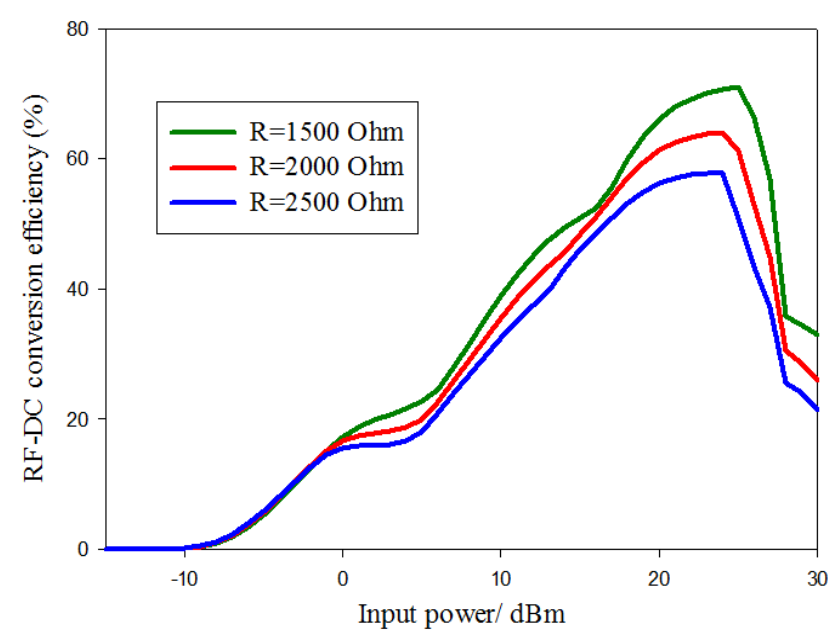

Figure 13. Simulation result of rectifier efficiency versus input Power.

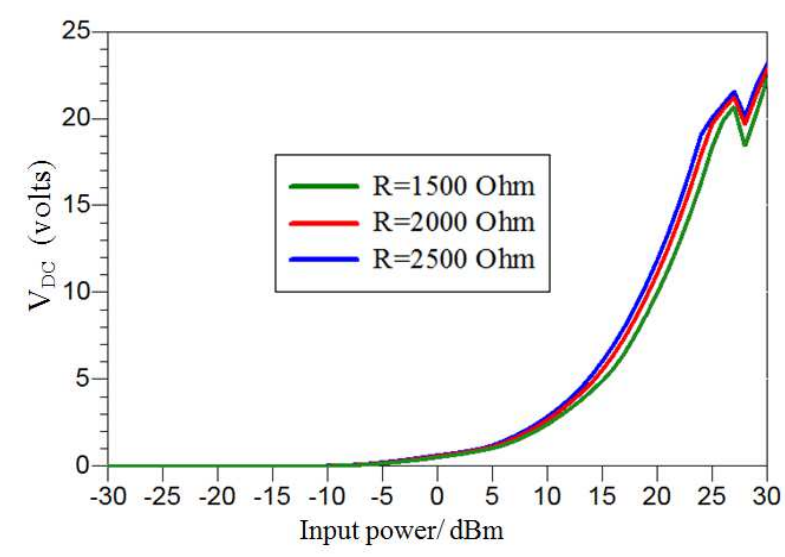

Figure 14. Simulation result of DC Output Voltage versus input Power.

\section{Conclusion}

An efficient rectenna design for Microwave Power Transmission systems at $2.45 \mathrm{GHz}$ has been proposed and analyzed using an analysis approach into its components using simulation methods. The developed design presents good features which makes it a suitable candidate to be used in a WPTs. The design provides a high directivity of its receiving antenna which maintains a circular polarization due to the good axial ratio obtained and also the good radiation efficiency that can reach more than $98 \%$ at the operating frequency. Further the enhanced RF-to-DC conversion efficiency of about $71 \%$ of the microstrip rectifier which exhibits an output DC voltage of $10 \mathrm{~V}$ where the input power level is around $20 \mathrm{dBm}$ with an optimum load of $1500 \Omega$ are a good features which provides that the developed design has the capability and the potential to be investigated for power supply using microwave power transmission at high power densities for RFID systems, WSN and future ubiquitous systems.

\section{Acknowledgment}

The authors would like acknowledge the contribution of the professor Mohamed Latrach from ESEO of Angers in France and the professor Jamal Zbitou from LMEET laboratory of FPK/FSTS of Hassan the first university in Morocco for their technical support in this research. The authors are also grateful to the anonymous reviewers for their constructive and helpful comments and suggestion.

\section{References}

[1] Riviere, S., F. Alicalapa, A. Douyere, and J.-D. Lan Sun Luk,"A compact rectenna device at low power level" Progress In Electromagnetic Research C, Vol. 16, 137-146, 2010.

[2] P. Koert, J. Cha, and M. Machina, "35 and $94 \mathrm{GHz}$ rectifying antenna systems," in SPS 91-Power from Space Dig., Paris, France, Aug. 1991, pp. 541-547.

[3] W.-H. Tu, S.-H. Hsu, and K. Chang, "Compact 5.8-GHz rectenna using stepped-impedance dipole antenna," IEEE Antenna and Wireless Propagation Letters, vol. 6, pp. 282-284, 2007. 
[4] Y.-J. Ren, M.-Y. Li, and K. Chang, "35 GHz rectifying antenna for wireless power transmission," Electronics Letters 24th, vol. 43, no.11, pp., May 2007.

[5] D. PavoneA. Buonanno, M. D'Urso, and F. D. Corte "Design considerations for radio frequency energy harvesting devices" Progress In Electromagnetics Research B, Vol. 45, 19-35, 2012.

[6] G. Monti F. Congedo, D. De Donno, and L. Tarricone "Monopole-based rectenna for microwave energy harvesting of UHF RFID systems" Progress In Electromagnetics Research C, Vol. 31, 109-121, 2012.

[7] J. Zbitou, M. Latrach, and S. Toutain, "Hybrid rectenna and monolithic integrated zero-bias microwave rectifier," IEEE Trans. Microw. Theory Tech., vol. 54, no. 1, pp. 147-152, J an. 2006.

[8] Wen Huang, Biao Zhang, Xing Chen, Kama Huang, and Changjun Liu "study on an s-band rectenna array for wireless microwave power transmission" Progress In Electromagnetics Research, Vol. 135, 747-758, 2013.

[9] G. Vera, A. Georgiadis, A. Collado, and S. Via, "Design of a $2.45 \mathrm{GHz}$ rectenna for electromagnetic (EM) energy scavenging," in IEEE Radio Wireless Symp., 2010, pp. 61-64.

[10] Erez Falkenstein, Michael Roberg, and Zoya Popovi "LowPower Wireless Power Delivery" IEEE Transactions on microwave theory and techniques 0018-9480/2012.

[11] Heikkinen, J. and M. Kivikoski," Low-profile circularly polarized rectifying antenna for wireless power transmission at $5.8 \mathrm{GHz}$," IEEE Microw. And Wirel. Compon. Lett. Vol. 14, No. 4, Apr. 2004

[12] Ali, M., G. Yang, and R. Dougal, "A new circularly polarized rectenna for wireless power transmission and data communication," IEEE Antenn. Wirel. Propag. Lett., Vol. 4, 205-208, 2005.

[13] K.-L. Wong, C.-C. Huang, and W.-S. Chen, "Printed ring slot antenna for circular polarization," IEEE Trans. Antennas Propagat., vol. 50, pp.75-77, Jan. 2002.

[14] M. K. Fries and R. Vahldieck, "Uniplanar circularly polarized slot-ring antenna architectures," Radio Sci., vol. 38, no. 2, pp. VIC5/1-VIC5/10,March/April 2003.

[15] B. Strassner and K. Chang, "5.8-GHz circularly polarized rectifying antenna for wireless microwave power transmission," IEEE Trans. Microwave Theory Tech., vol. 50, no. 8, pp. 1870-1876, August 2002.
[16] M. A Sennouni, J. Zbitou, A. Benaissa, A. Tribak, O. Elmrabet, M. Latrach" Development of a New Slit-Slotted Shaped Microstrip Antenna Array for Rectenna Application" Journal of Emerging Technologies in Web Intelligence, Vol 6, No 1, 49-53, Feb 2014.

[17] Computer Simulation Technology, www.cst.com

[18] T. Q. V. Hoang, A. Douyere, J-L. Dubard J-D. Lan Sun Luk "TLM Design of a compact PIFA rectenna", IEEE 2011.

[19] Xue-Xia Yang, Chao Jiang, Atef Z. Elsherbeni, Fan Yang, YeQing Wang "A Novel Compact Printed Rectenna for Communication Systems", IEEE 2012.

[20] Y. J. Ren and K. Chang, "5.8-GHz Circularly Polarized DualDiode Rectenna and Rectenna Array for Microwave Power Transmission," IEEE Trans. Microw. Theory and Tech., vol. 54, no. 4, pp. 1495-1502, Apr. 2006.

[21] Jingwei Zhang, Yi Huangand Ping Cao "A Wideband Cross Dipole Rectenna for RF Wireless Harvesting" proceeding of (EUCAP 2013), IEEE 2013.

[22] Ugur Olgun, Chi-Chih Chen and John L. Volakis "Wireless Power Harvesting with Planar Rectennas for 2.45 GHz RFIDs" proceeding of URSI international symposium on electromagnetic theory, IEEE 2010.

[23] N. M. Din, C. K. Chakrabarty, A. Bin Ismail, K. K. A. Devi, and W.-Y. Chen "design of RF energy harvesting system for energizing low power devices" Progress in Electromagnetics Research, Vol. 132, 49-69, 2012.

[24] Hewlett Packard, Technical Data "Surface Mount Microwave Schottky Detector Diodes".

[25] H. Takhedmit, B. Merabet, L. Cirio, B. Allard, F. Costa, C. Vollaire, and O. Picon, "Design of a $2.45 \mathrm{GHz}$ rectenna using a global analysis technique," EuCAP 2009, pp. 2321-2325, March 2009, Berlin.

[26] C. S. Ong, M. F. Karim, L. C. Ong, T. M. Chiam A. Alphones "A Compact 2x2 Circularly Polarized Antenna Array for Energy Harvesting" in proceedings of Asia-Pacific Microwave Conference, IEEE 2010.

[27] M. F. Ismail, M. K. A. Rahim, M. R. Hamid, H. A. Majid and M. F. M. Yusof "Compact Dual-fed Slotted Circular Polarization Antenna with Reflector for RF Energy Harvesting" proceeding of 7 th European Conference on Antenna and propagation (EuCAP) IEEE 2013. 